\title{
Antioxidant responses to an acute ultra-endurance exercise: impact on DNA stability and indications for an increased need for nutritive antioxidants in the early recovery phase
}

\author{
Oliver Neubauer ${ }^{1}$, Stefanie Reichhold ${ }^{1}$, Lukas Nics ${ }^{1}$, Christine Hoelzl ${ }^{2}$, Judit Valentini ${ }^{1}$, \\ Barbara Stadlmayr ${ }^{1}$, Siegfried Knasmüller ${ }^{2}$ and Karl-Heinz Wagner ${ }^{1}$ \\ ${ }^{1}$ Department of Nutritional Sciences, Emerging Field Oxidative Stress and DNA Stability, Faculty of Life Sciences, \\ University of Vienna, Althanstraße 14, 1090 Vienna, Austria \\ ${ }^{2}$ Environmental Toxicology Group, Institute of Cancer Research, Medical University of Vienna, Borschkegasse 8A, \\ 1090 Vienna, Austria
}

(Received 9 December 2009 - Revised 9 April 2010 - Accepted 12 April 2010 - First published online 19 July 2010)

Antioxidant requirements have neither been defined for endurance nor been defined for ultra-endurance athletes. To verify whether an acute bout of ultra-endurance exercise modifies the need for nutritive antioxidants, we aimed (1) to investigate the changes of endogenous and exogenous antioxidants in response to an Ironman triathlon; (2) to particularise the relevance of antioxidant responses to the indices of oxidatively damaged blood lipids, blood cell compounds and lymphocyte DNA and (3) to examine whether potential time-points of increased susceptibility to oxidative damage are associated with alterations in the antioxidant status. Blood that was collected from forty-two well-trained male athletes $2 \mathrm{~d}$ pre-race, immediately post-race, and 1, 5 and $19 \mathrm{~d}$ later was sampled. The key findings of the present study are as follows: (1) Immediately post-race, vitamin $\mathrm{C}, \alpha$-tocopherol, and levels of the Trolox equivalent antioxidant capacity, the ferric reducing ability of plasma and the oxygen radical absorbance capacity (ORAC) assays increased significantly. Exercise-induced changes in the plasma antioxidant capacity were associated with changes in uric acid, bilirubin and vitamin C. (2) Significant inverse correlations between ORAC levels and indices of oxidatively damaged DNA immediately and $1 \mathrm{~d}$ post-race suggest a protective role of the acute antioxidant responses in DNA stability. (3) Significant decreases in carotenoids and $\gamma$-tocopherol $1 \mathrm{~d}$ post-race indicate that the antioxidant intake during the first $24 \mathrm{~h}$ of recovery following an acute ultra-endurance exercise requires specific attention. Furthermore, the present study illustrates the importance of a diversified and well-balanced diet to maintain a physiological antioxidant status in ultra-endurance athletes in reference to recommendations.

Ironman triathlon: Plasma antioxidant defences: Oxidative DNA damage: Antioxidant requirements

Due to extensive research done in the past three decades, it is well known that strenuous aerobic exercise can result in an increase of reactive oxygen and nitrogen species (RONS) ${ }^{(1)}$. However, there is now sufficient evidence that RONS are not only damaging agents, but also act as signalling molecules to initiate exercise adaptations ${ }^{(2)}$ and to regulate muscle function $^{(1)}$. In this context, most recent investigations indicated that high dosages of antioxidants can blunt cellular training adaptations $^{(3)}$ and health-promoting effects ${ }^{(4)}$. These findings have contributed important novel aspects to the ongoing debate whether supplementation with antioxidants is useful in terms of preventing or minimising oxidative stress and damage in response to strenuous exercise or detrimental by interfering with beneficial physiological effects of exercise training ${ }^{(5)}$. Until now, the question of antioxidant requirements (both regarding certain antioxidant micronutrients and regarding dosages of these antioxidants) for endurance athletes in general and the hypothetical need for additional antioxidant supplementation has not been discussed sufficiently ${ }^{(6)}$. Among endurance athletes, Ironman/long-distance triathletes are an exceptional group to investigate due to their training and performance level, physiological characteristics ${ }^{(7)}$ and nutritional challenges ${ }^{(8)}$, and urgently, to assess probable harmful effects of ultra-endurance sports in general. Some empirical and epidemiological data suggest that an extraordinarily high volume of exercise is associated with an increased risk of developing $\mathrm{CVD}^{(9)}$ due to cumulative oxidative stress as one of the main potential mechanisms ${ }^{(10)}$. Therefore, it is of particular importance to determine antioxidant requirements for this continually growing population of athletes training for and competing in races with durations of several hours. Noteworthily, outcomes of studies that examined high-dosed antioxidant supplementation explicitly in competitors of long-distance triathlons or ultra-marathon

Abbreviations: AOPP, advanced oxidation protein products; FRAP, ferric reducing ability of plasma; ORAC, oxygen radical absorbance capacity; RONS, reactive oxygen and nitrogen species; TEAC, Trolox equivalent antioxidant capacity.

* Corresponding author: Dr O. Neubauer, fax +4314277 9549, email oliver.neubauer@univie.ac.at 
runs are quite controversial, showing either beneficial ${ }^{(11)}$, adverse (i.e. pro-oxidant) effects ${ }^{(12,13)}$ or no effects ${ }^{(14)}$ on the changes in the oxidative stress markers. Thus, instead of an interventional supply with antioxidants, it is reasonable to consider the nutritional conditions before, during and after such a competition. Therefore, when particularly addressing the question whether antioxidant requirements are modified in response to an acute ultra-endurance exercise, attention has to be paid to the antioxidant intake during such a competition and to the changes in the antioxidant status in response to this type of exercise. Furthermore, when drawing the focus on antioxidant requirements for this group of endurance athletes in general, it is important to take potential influencing factors, such as training-induced adaptations in the endogenous antioxidant system, interactions between endogenous antioxidant defences and nutritive antioxidants and their dietary pattern, into account.

The present data are part of a larger study that aimed to get a broader picture of exercise-induced skeletal muscle damage, inflammatory and immunological alterations ${ }^{(15)}$, myocardial stress $^{(16)}$, oxidative stress ${ }^{(17)}$, genome stability ${ }^{(18,19)}$ and the relationship of DNA damage with inflammatory responses ${ }^{(20)}$ in a large cohort of participants of an Ironman triathlon ( $n$ 42). Within the scope of the currently presented part of the present study, particular focus was drawn on the modifications in the plasma concentrations of endogenous low-molecular weight (i.e. uric acid, bilirubin and plasma protein) and nutritive antioxidants (i.e. $\alpha$ - and $\gamma$-tocopherol, carotenoids and vitamin $\mathrm{C}$ ) after the Ironman race. Hence, the main objectives of this part of the present study are as follows: on the one hand, we aimed to particularise the relevance of the antioxidant responses to the previously reported indices of lipid peroxidation, protein oxidation and DNA stability in blood plasma and in circulating lymphocytes, respectively ${ }^{(17,19)}$. On the other hand, we intended to determine whether there are distinct timepoints after an ultra-endurance exercise in which athletes are particularly vulnerable to oxidative damage. Importantly, we expected that the verification whether such hypothesised time-points are associated with alterations in the antioxidant status in plasma might provide indications for an increased requirement of nutritive antioxidants and a potential need for supplementation in certain phases after intense and very prolonged exercise. In the attempt to verify the time course of recovery, these responses were monitored until $19 \mathrm{~d}$ after the race, and thus longer than any other study in this context. Finally, it was important to examine oxidative stress and antioxidant responses under 'authentic' race conditions, i.e. without any nutritional intervention, but with careful assessment of the intake of antioxidant vitamins and carotenoids during the race and during the early recovery period.

\section{Experimental methods}

\section{Study design and subjects}

The design of the study has been described previously ${ }^{(15,17)}$. Briefly, it comprised forty-eight non-professional, well-trained male triathletes, who participated in the 2006 Ironman Austria. The study was conducted according the guidelines of the Declaration of Helsinki, and the study was approved by the Ethics Committee of the Medical University of Vienna.
All the study participants were informed about the purpose and the risks of the investigation before they provided written informed consent. Forty-two of the subjects completed the study, and were included in the statistical analysis. The physiological characteristics of the study participants (assessed using a cycle ergometer 3 weeks before the Ironman triathlon) in addition to their training history are summarised in Table 1 (for more detailed information, see Neubauer et al. ${ }^{(15)}$ and Neubauer et $\left.a l .{ }^{(17)}\right)$. All the participants were physically fit, non-smokers, within a normal range of BMI, and free of acute and chronic illnesses. They were asked to provide extensive information on their medical and health status, their training schedule and their nutritional habits including a supplementation and an FFQ. The subjects did not take any medication 6 weeks before the Ironman race until the end of the study. Crucially, as an inclusion criterion for participating in the study, they were instructed to avoid antioxidant supplementation at larger doses, i.e. more than $100 \%$ of $\mathrm{RDA}^{(21)}$ (in addition to their normal dietary antioxidant intake) throughout this period (except during the race, see below). Consequently, they did not consume more than $60 \mathrm{mg}$ of vitamin $\mathrm{C}, 10 \mathrm{mg}$ of $\alpha$-tocopherol, $4 \mathrm{mg}$ of $\beta$-carotene (which equates the upper level of the regionspecific recommendation range ${ }^{(22)}$ since there is no RDA value available), $70 \mu \mathrm{g}$ of $\mathrm{Se}, 15 \mathrm{mg}$ of $\mathrm{Zn}$ or any other antioxidant in the form of supplements. Compliance was controlled by self-reported documentation of the athletes as well as by measurement of the plasma concentrations of most of these antioxidants. Only during the Ironman race, the dietary intake (including antioxidant-fortified beverages and food) was ad libitum, but the quantities of the intake of nutrients were recorded both by the athletes themselves via a $24 \mathrm{~h}$ dietary recall (see below) and by the helpers who monitored their food intake at each of the supply stations of the race. The Ironman triathlon (consisting of $3.8 \mathrm{~km}$ swimming, $180 \mathrm{~km}$ cycling and $42.2 \mathrm{~km}$ running) took place in Klagenfurt, Austria, on 16 July 2006 on a warm $\left(27 \cdot 2^{\circ} \mathrm{C}\right)$ and dry day ${ }^{(15,17)}$. Blood samples were collected $2 \mathrm{~d}$ prerace, immediately post-race (within $20 \mathrm{~min}$ ), and 1,5 and $19 \mathrm{~d}$ post-race. Except the Ironman race itself, the study participants abstained from intense exercise $48 \mathrm{~h}$ before each

Table 1. Main characteristics of the subjects and their performance in the Ironman triathlon

(Mean values and standard deviations)

\begin{tabular}{|c|c|c|}
\hline & Mean & SD \\
\hline Age (years) & $35 \cdot 3$ & $7 \cdot 0$ \\
\hline Height $(\mathrm{cm})$ & $180 \cdot 6$ & $5 \cdot 6$ \\
\hline Weight (kg) & $75 \cdot 1$ & $6 \cdot 4$ \\
\hline BMI $\left(\mathrm{kg} / \mathrm{m}^{2}\right)$ & $23 \cdot 0$ & $1 \cdot 2$ \\
\hline Cycling $\mathrm{VO}_{2}$ peak $(\mathrm{ml} / \mathrm{kg}$ per $\mathrm{min})$ & $56 \cdot 6$ & $6 \cdot 2$ \\
\hline Peak power output ( $\left.W_{\text {peak }}\right)$ & $357 \cdot 9$ & $50 \cdot 0$ \\
\hline Individual anaerobic threshold ( $W$ ) & $219 \cdot 4$ & 43.5 \\
\hline \multicolumn{3}{|l|}{$\begin{array}{l}\text { Training over a period of } 6 \text { months } \\
\text { before the Ironman triathlon }\end{array}$} \\
\hline Weekly net endurance exercise time (h/week) & $10 \cdot 7$ & $2 \cdot 6$ \\
\hline \multicolumn{3}{|l|}{ Performance in the Ironman triathlon } \\
\hline Total race time (h:min:s) & $10: 51: 52$ & 01:01:22 \\
\hline \multicolumn{3}{|l|}{$\begin{array}{l}\text { Training after the completion of the } \\
\text { Ironman triathlon until } 19 \mathrm{~d} \text { post-race }\end{array}$} \\
\hline Weekly net endurance exercise time (h/week) & $4 \cdot 2$ & 2.4 \\
\hline
\end{tabular}


blood sampling, and they had fasted overnight before the $2 \mathrm{~d}$ pre-race blood sampling, and 5 and $19 \mathrm{~d}$ post-race blood sampling. After the Ironman race until the end of the study, the subjects underwent only moderate ('recovery') training (Table 1).

\section{Assessment of the intake of antioxidant vitamins and} carotenoids during the race and within the first $24 \mathrm{~h}$ post-race

Before each blood sampling, the athletes were required to provide $24 \mathrm{~h}$ dietary recalls. The main focus was on the intake of antioxidant vitamins and carotenoids during the race and within the first $24 \mathrm{~h}$ post-race. Analyses were performed based on the German/Austrian Food database BLS II (BGVV, Berlin Germany and ÖLS, Vienna, Austria). Special food and beverage items used by the athletes (e.g. carbohydrate bars and sports drinks) were added to the database.

\section{Plasma concentrations of nutritive antioxidants and antioxidative biochemical substances}

Plasma concentrations of $\alpha$ - and $\gamma$-tocopherol, $\beta$-carotene, lutein/zeaxanthin, cryptoxanthin and lycopene were measured with HPLC (Ultimate 3000; Dionex Corporation, Vienna, Austria). Plasma vitamin $\mathrm{C}$ was analysed photometrically. Plasma concentrations of total protein, total bilirubin and uric acid were measured using an automatic analyser (Vitro DT 60 II module; Ortho-clinical Diagnostics, Neckargemünd, Germany).

\section{Plasma concentrations of lipid peroxidation and protein} oxidation markers

As reported in a previous publication ${ }^{(17)}$ and described before $^{(23)}$, both malondialdehyde and conjugated dienes were measured with HPLC (pump, detector and integrator; Hitachi, Tokyo, Japan and column; Merck, Darmstadt, Germany). Oxidised LDL concentrations and advanced oxidation protein products (AOPP) were determined using an ELISA kit (Mercodia AB, Uppsala, Sweden) and a colorimetric assay kit (Immundiagnostik AG, Bensheim, Germany), respectively ${ }^{(17)}$.

\section{Total antioxidant capacity of plasma}

As reported previously ${ }^{(17,19)}$, three approaches were applied to assess the total antioxidant capacity of plasma: the Trolox equivalent antioxidant capacity (TEAC), the ferric reducing ability of plasma (FRAP) and the oxygen radical absorbance capacity (ORAC) assays. The first two assays are used to measure the ability of a substance to transfer one electron to reduce compounds such as radicals or metals, while the ORAC assay is based on the ability of a substance to quench free radicals by hydrogen donation ${ }^{(24)}$. Briefly, the TEAC assay is used to measure the ability of antioxidants in the plasma to delay the oxidation of the 2,2-azinobis (3-ethylbenzthiazoline-6-sulphonic acid) radical. The assay was performed as described previously ${ }^{(25)}$. The FRAP assay was conducted according to the protocol of Benzie \& Strain ${ }^{(26)}$ with modifications developed by the same authors $^{(27)}$. The protocol reflects the capacity of a substance to reduce the ferric tripyridyltriazine $\left(\mathrm{Fe}^{3+}\right.$-tripyridyl triazine) to the ferrous and intense blue-coloured $\left(\mathrm{Fe}^{2+}-\right)$ tripyridyl triazine complex. Finally, the ORAC assay was carried out as described by Ou et al. ${ }^{(28)}$ and Huang et $a{ }^{(29)}$. This assay is based on the antioxidant inhibition of peroxyl radicals, which are generated by plasma antioxidants $2,2^{\prime}$-azobis (2-amidinopropane)-dihydrochloride.

\section{DNA damage in lymphocytes}

As described by Reichhold et al. ${ }^{(19)}$ and Wagner et al. ${ }^{(30)}$, the single-cell gel electrophoresis (comet) assay including the use of lesion-specific enzymes endonuclease III and formamidopyrimidine glycosylase was applied to assess single- and double-strand breaks (determined as the percentage of DNA in the tail), and oxidised purines and pyrimidines, respectively. Twenty-eight subjects were randomly selected for the single-cell gel electrophoresis assay, and lymphocytes were harvested from the blood samples by centrifugation (for details, see Reichhold et al. $^{(19)}$ ). The single-cell gel electrophoresis assays were conducted according to the methods described by Tice et $a l^{(31)}$, and oxidative DNA base damage was assessed on the basis of the protocols of Collins et al. ${ }^{(32)}$.

\section{Haematological profile}

The haematological profile was assessed using a MS4 Hematology 3-Part-Differential-Analyzer (Melet Schloesing Laboratories, Maria Enzersdorf, Austria). Exercise-induced percentage changes in plasma volume were calculated ${ }^{(33)}$ until $5 \mathrm{~d}$ post-race to assess potential alterations (i.e. an initial contraction followed by a potential expansion) of plasma volume, which may persist for 3-5d after the cessation of demanding exercise ${ }^{(34)}$. All the results were adjusted for these changes, except for the plasma antioxidant capacity, and ratios of oxidised LDL-LDL and AOPP-total protein. For the determination of these markers, we used the data uncorrected for changes in plasma volume to consider their actual concentration to which the body responds. Correspondingly, 'real' plasma concentrations of antioxidants (i.e. without adjustment for plasma volume changes) were used when potential associations with the plasma antioxidant capacity were examined. In the present study, the adjustment for plasma volume changes had no significant impact on the statistical results.

\section{Data analysis}

All statistical analyses were performed using SPSS 15.0 for Windows (SPSS, Chicago, IL, USA). Details of the data analysis have been presented previously ${ }^{(15,17,35)}$. The main effect of time was obtained using the repeated-measures ANOVA. To examine significant relationships, Pearson's (for normally distributed data) or Spearman's (for nonnormally distributed data) correlation was used. Furthermore, the subjects were divided into four percentile groups by the plasma concentrations and intakes of the antioxidant vitamins and $\beta$-carotene. One-factorial ANOVA and post hoc analyses with Scheffé's test were applied to assess whether differences in the changes of oxidative stress markers, total antioxidant capacity and DNA damage in lymphocytes were associated with the percentile distribution. The significance level was 
Table 2. Total energy intake, intake of macronutrients, antioxidant vitamins and $\beta$-carotene during and within the first $24 \mathrm{~h}$ after the Ironman race

(Mean values and standard deviations)

\begin{tabular}{|c|c|c|c|c|c|c|c|c|}
\hline & \multicolumn{2}{|c|}{$\begin{array}{l}\text { During the } \\
\text { cycle split }\end{array}$} & \multicolumn{2}{|c|}{$\begin{array}{l}\text { During the } \\
\text { run split }\end{array}$} & \multicolumn{2}{|c|}{$\begin{array}{c}\text { Total intake } \\
\text { during the race }\end{array}$} & \multicolumn{2}{|c|}{$\begin{array}{l}\text { Within } 24 \mathrm{~h} \\
\text { post-race }\end{array}$} \\
\hline & Mean & SD & Mean & SD & Mean & SD & Mean & SD \\
\hline Energy (kJ) & 8974 & 3821 & 5246 & 2905 & 14220 & 5342 & 18848 & 10868 \\
\hline Carbohydrate (g) & 480 & 217 & 237 & 158 & 753 & 297 & 564 & 375 \\
\hline Fat $(\mathrm{g})$ & 12 & 10 & 2 & 6 & 14 & 13 & 158 & 84 \\
\hline Protein (g) & 31 & 20 & 27 & 12 & 59 & 26 & 144 & 70 \\
\hline Vitamin C (mg) & 289 & 153 & 103 & 113 & 393 & 219 & 244 & 248 \\
\hline$\alpha$-Tocopherol (mg) & 81 & 47 & 32 & 31 & 113 & 59 & $10 \cdot 1$ & 5.7 \\
\hline$\beta$-Carotene $(\mathrm{mg})$ & $6 \cdot 8$ & 13.5 & $2 \cdot 3$ & $6 \cdot 3$ & $9 \cdot 1$ & 17.9 & 2.7 & $2 \cdot 3$ \\
\hline
\end{tabular}

set at a $P<0.05$, and is reported as $P<0.05,<0.01$ and $<0.001$. Significance levels are quoted two sided.

\section{Results}

\section{Race results}

The study participants completed the Ironman triathlon in 10 (SD 1)h 52 (SD 1) min. Of forty-eight subjects, three subjects dropped out of the Ironman race due to self-reported fatigue symptoms, while three other subjects could not participate in one or more blood samplings.

Intake of vitamin $C, \alpha$-tocopherol and $\beta$-carotene during the Ironman race and within the first $24 \mathrm{~h}$ post-race

The average intake of vitamin $\mathrm{C}, \alpha$-tocopherol and $\beta$-carotene was 393 (SD 219), 113 (SD 59) and 9.1 (SD 17.9) mg during the Ironman race, and 244 (SD 248), 10.1 (SD 5.7) and 2.7 (SD $2 \cdot 3$ ) $\mathrm{mg}$ within the first $24 \mathrm{~h}$ after the race, respectively. Data pertaining to the consumption of these antioxidants are summarised in Table 2.

\section{Plasma concentrations of nutritive antioxidants}

The time courses of plasma concentrations of vitamin $\mathrm{C}$, $\alpha$-tocopherol, $\gamma$-tocopherol and carotenoids in response to the Ironman race are summarised in Table 3. An immediate significant increase in response to the triathlon was observed in both vitamin $\mathrm{C}$ and $\alpha$-tocopherol $(+54$ and $+18 \%$, respectively; for both $P<0 \cdot 01$ ). One day post-race, vitamin $\mathrm{C}$ concentrations decreased to values that were similar to the pre-race values, whereas other antioxidant nutrient concentrations dropped significantly to below the pre-race concentrations: $\gamma$-tocopherol $(-25 \% ; \quad P<0.01)$, lutein/ zeaxanthin $(-15 \% ; P<0.05)$, cryptoxanthin $(-8 \% ; P<0.05)$ and $\beta$-carotene $(-15 \% ; P<0 \cdot 01)$. The decrease in $\alpha$-tocopherol $1 \mathrm{~d}$ post-race $(-4 \%)$ did not reach statistical significance. Most nutritive antioxidant concentrations were similar to the pre-race values, except for vitamin C, 5 and $19 \mathrm{~d}$ post-race. Vitamin $C$ level increased to above the pre-race levels $5 \mathrm{~d}$ post-race $(+21 \% ; P<0.01)$, and remained elevated $19 \mathrm{~d}$ post-race $(+24 \% ; P<0 \cdot 01)$.

\section{Plasma concentrations of endogenous antioxidative compounds}

Plasma levels of uric acid significantly $(P<0.001)$ increased immediately after the triathlon $(+49 \%)$. Subsequently, the uric acid concentrations gradually declined, but remained significantly $(P<0.001)$ elevated at all the time-points $(P=0.001$ for $19 \mathrm{~d}$ post-race $) v$. the pre-race values ${ }^{(17)}$. Plasma total bilirubin was significantly $(P<0 \cdot 001)$ higher

Table 3. Plasma concentrations of endogenous and nutritive antioxidants (Mean values and standard deviations)

\begin{tabular}{|c|c|c|c|c|c|c|c|c|c|c|c|}
\hline & \multicolumn{2}{|c|}{ Pre-race } & \multicolumn{2}{|c|}{ Post-race } & \multicolumn{2}{|c|}{$1 \mathrm{~d}$ post-race } & \multicolumn{2}{|c|}{$5 \mathrm{~d}$ post-race } & \multicolumn{2}{|c|}{19 d post-race } & \multirow[b]{2}{*}{ Time effect $(P)$} \\
\hline & Mean & SD & Mean & SD & Mean & SD & Mean & SD & Mean & SD & \\
\hline \multicolumn{12}{|l|}{ Endogenous antioxidants } \\
\hline Uric acid $(\mu \mathrm{mol} / \mathrm{l})$ & 311 & 54 & $465^{\star \star \star}$ & 92 & $422^{\star \star \star}$ & 63 & $338^{\star \star \star}$ & 62 & $328^{\star \star *}$ & 53 & $<0.001$ \\
\hline Total bilirubin $(\mu \mathrm{mol} / \mathrm{l})$ & 9.7 & 53 & $13 \cdot 6^{\star * *}$ & 5.4 & $16 \cdot 0^{\star \star *}$ & $8 \cdot 0$ & $6 \cdot 5^{\star \star \star}$ & 3.8 & $9 \cdot 3$ & $5 \cdot 5$ & $<0.001$ \\
\hline Total plasma protein $(\mathrm{g} / \mathrm{l})$ & $7 \cdot 6$ & 0.3 & $7 \cdot 9^{\star \star}$ & 0.7 & $7 \cdot 0^{\star \star \star}$ & 0.4 & $7 \cdot 0^{\star \star \star}$ & 0.6 & $7 \cdot 0$ & 0.5 & $<0.001$ \\
\hline \multicolumn{12}{|l|}{ Nutritive antioxidants } \\
\hline Vitamin C ( $\mu \mathrm{mol} / \mathrm{l})$ & $66 \cdot 6$ & $13 \cdot 0$ & $102 \cdot 8^{\star *}$ & $26 \cdot 6$ & $68 \cdot 2$ & $13 \cdot 2$ & $80 \cdot 9^{\star *}$ & $15 \cdot 2$ & $82 \cdot 8^{\star *}$ & $14 \cdot 0$ & $<0.001$ \\
\hline$\alpha$-Tocopherol $(\mu \mathrm{mol} / \mathrm{l})$ & $22 \cdot 6$ & $7 \cdot 3$ & $26 \cdot 6^{\star \star}$ & $7 \cdot 0$ & $21 \cdot 8$ & $6 \cdot 1$ & $22 \cdot 5$ & $5 \cdot 8$ & $21 \cdot 8$ & $5 \cdot 8$ & $<0.001$ \\
\hline$\gamma$-Tocopherol $(\mu \mathrm{mol} / \mathrm{l})$ & $1 \cdot 29$ & 0.67 & $1 \cdot 22$ & 0.60 & $0.97^{\star *}$ & 0.38 & $1 \cdot 29$ & 0.59 & $1 \cdot 29$ & 0.48 & $<0.01$ \\
\hline$\beta$-Carotene $(\mu \mathrm{mol} / \mathrm{l})$ & 0.88 & 0.36 & 0.88 & 0.36 & $0 \cdot 75^{\star *}$ & 0.31 & 0.85 & 0.36 & 0.84 & 0.34 & 0.001 \\
\hline Lutein/zeaxanthin $(\mu \mathrm{mol} / \mathrm{l})$ & 0.45 & 0.15 & 0.45 & 0.15 & $0 \cdot 40^{\star}$ & 0.13 & 0.42 & $0 \cdot 16$ & 0.40 & 0.15 & $<0.05$ \\
\hline Cryptoxanthin $(\mu \mathrm{mol} / \mathrm{l})$ & $0 \cdot 12$ & 0.07 & $0 \cdot 11^{*}$ & 0.06 & $0 \cdot 11^{*}$ & 0.06 & $0 \cdot 12$ & 0.06 & $0 \cdot 13$ & 0.08 & $<0.01$ \\
\hline Lycopene $(\mu \mathrm{mol} / \mathrm{l})$ & 0.24 & 0.13 & 0.26 & 0.12 & 0.22 & 0.10 & 0.25 & 0.10 & 0.24 & 0.10 & 0.001 \\
\hline
\end{tabular}

Mean values were significantly different from the pre-race value: ${ }^{\star} P<0.05,{ }^{\star \star} P<0.01,{ }^{\star \star *} P<0.001$. 
immediately after the race $(+39 \%)$ than pre-race, increased further $1 \mathrm{~d}$ post-race $(+65 \%)$, and significantly $(P<0.001)$ declined below the pre-race concentrations $5 \mathrm{~d}$ post-race. Plasma total protein increased immediately after the Ironman race $(+4 \% ; P<0.01)$, whereas there was a significant decrease to below the pre-race values thereafter.

\section{Total antioxidant capacity of plasma}

The time course of the total plasma antioxidant capacity, assessed by TEAC, FRAP and ORAC assays, is shown in Fig. 1. Similar time courses were observed for all these assays. As described previously ${ }^{(17,19)}$, TEAC, FRAP and ORAC values increased significantly $(P<0.001)$ immediately after the Ironman race $(+48,+35$ and $+18 \%$, respectively). The values of all the assays remained significantly higher than the pre-race values until $1 \mathrm{~d}$ after the race $(+25,+24$ and $+11 \%$, respectively; $P<0.001$ for TEAC and FRAP, and $P<0.05$ for ORAC). Five days after the race, TEAC, FRAP and ORAC values had declined back to the baseline levels, and $19 \mathrm{~d}$ post-race, they remained similar to the pre-race values.

\section{Plasma concentrations of lipid peroxidation and protein} oxidation markers

For more detailed information on oxidative stress responses, the reader is referred to a previously published article $^{(17)}$. Briefly, except for oxidised LDL and the oxidised LDL-LDL ratio, all oxidative stress markers significantly increased in response to the Ironman triathlon. While conjugated dienes, AOPP and the ratio of AOPP to total protein peaked immediately post-race $(+91,+25$ and $+20 \%$, respectively; $P<0.01$ for conjugated dienes and AOPP, and $P<0.01$ for AOPP-total protein ratio), the increase of malondialdehyde reached statistical significance $1 \mathrm{~d}$ post-race ( $+9 \% ; \quad P<0.01$ ) (after correcting the values for the increase in plasma volume at this time-point). One day postrace, with the exception of oxidised LDL (which decreased) and oxidised LDL-LDL ratio (which tended to remain elevated), all oxidative stress markers were above the pre-race levels. Five days post-race, all the oxidative stress markers returned to the pre-race values, remaining at these concentrations $19 \mathrm{~d}$ post-race.

\section{DNA damage in lymphocytes}

As reported by Reichhold et al. ${ }^{(19)}$, a decrease of DNA strand breaks was observed immediately after the race. One day post-race, DNA migration due to strand breaks increased $(P<0.01)$, then returned to the pre-race levels $5 \mathrm{~d}$ post-race, and decreased further to below the initial levels $19 \mathrm{~d}$ post-race $(P<0 \cdot 01)$. Compared with the pre-race situation, no significant changes were observed in the levels of endonuclease III- and formamidopyrimidine glycosylase-sensitive sites throughout the entire monitoring period.
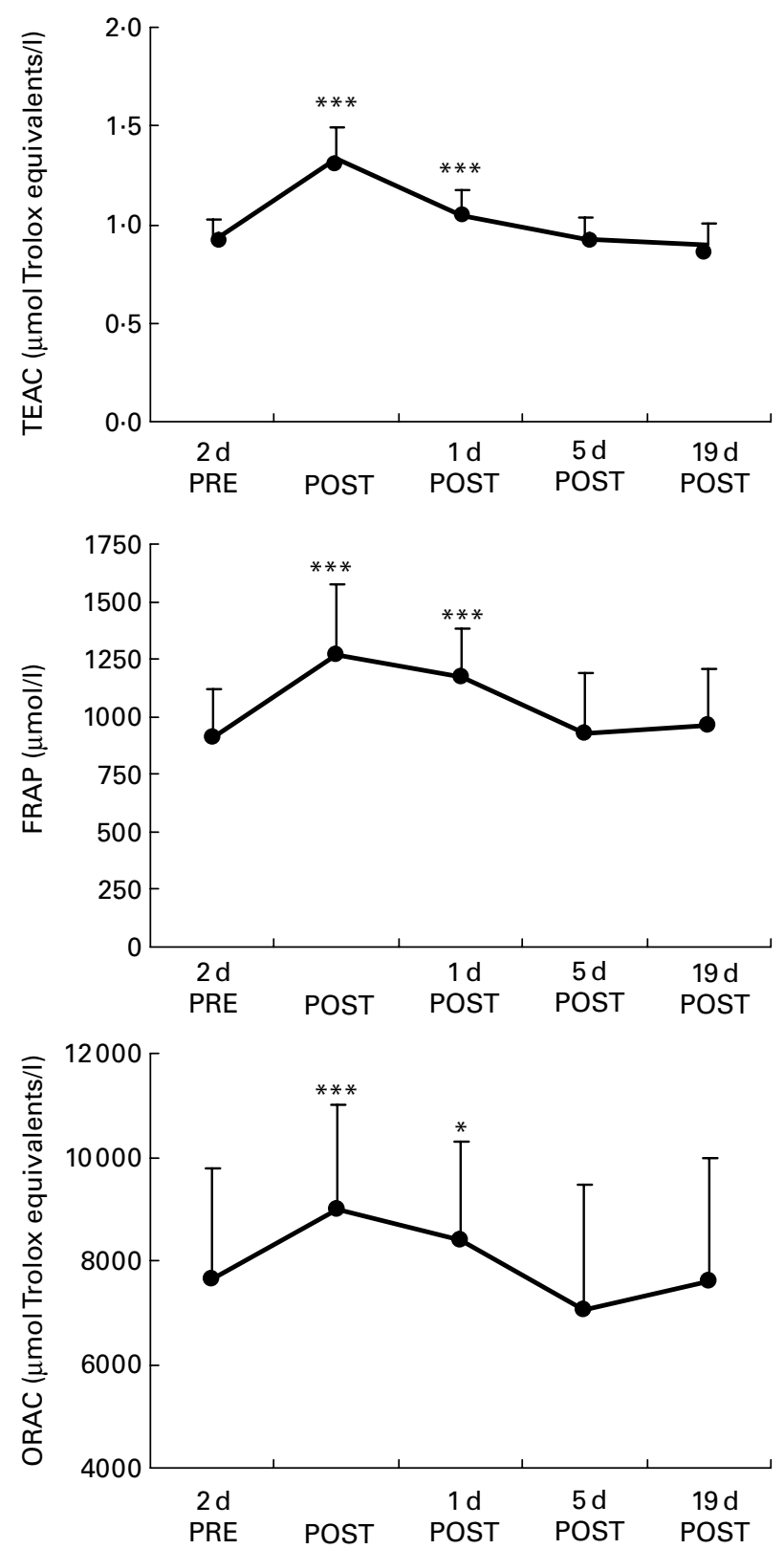

Fig. 1. Time course of the total plasma antioxidant capacity as assessed by the Trolox equivalent antioxidant capacity (TEAC), the ferric reducing ability of plasma (FRAP) and the oxygen radical absorbance capacity (ORAC) assays $2 \mathrm{~d}$ pre-race (PRE), immediately post-race, and 1, 5 and $19 \mathrm{~d}$ post-race (POST). Values are means and standard deviations are represented by vertical bars. Mean values were significantly different from the pre-race values: ${ }^{\star} P<0.05$, ${ }^{\star \star \star} P<0.001$.

Associations with plasma concentrations of nutritive and endogenous antioxidants, intake of antioxidant vitamins and carotenoids and plasma antioxidant capacity

Moderate to strong positive correlations were observed between the pre- and post-race changes in uric acid on the one side, and between ORAC $(r 0.36 ; P<0.05)$, TEAC ( $r$ 0.54; $P<0.001)$ and FRAP $(r 0.73$; and $P<0.001)$ on the other side. Furthermore, correlations were found between pre- and post-race changes in TEAC and in vitamin $\mathrm{C}$ 
( $r 0.33 ; P<0.05)$, pre- and post-race changes in FRAP and in total bilirubin $(r 0.42 ; P<0.01)$, and ORAC levels and total plasma protein concentrations pre-race $(r$ 0.44; $P<0.01)$, post-race $(r 0.46 ; P<0.01)$ and $5 \mathrm{~d}$ post-race ( $r \quad 0.39 ; P<0.05$ ). The dose-effect relationship logarithmic regression of the vitamin $\mathrm{C}$ intake during the race $v$. the post-race plasma concentration is shown in Fig. 2.

\section{Associations with nutritive and endogenous antioxidants,} oxidative stress markers and DNA damage

Significant positive correlations were observed between DNA migration due to endonuclease III-sensitive sites and the ORAC values immediately post-race $(r-0.54 ; P<0.01)$ and also $1 \mathrm{~d}$ post-race $(r-0.65 ; P<0.05)(\text { Fig. } 3)^{(20)}$. Furthermore, no associations were found between antioxidant responses on the one side, and between lipid peroxidation and protein oxidation markers on the other side.

Results based on the group distributions into percentiles by plasma concentrations and intakes of the antioxidant vitamins and $\beta$-carotene and effects of supplementation

No associations with oxidative stress markers, total antioxidant capacity and endpoints of DNA damage were observed on the basis of percentile distribution by the plasma concentrations and intakes of vitamin $C, \alpha$-tocopherol and $\beta$-carotene. Furthermore, there was no significant difference either in the pre-race values or in the post-race values of any antioxidant variable between the study participants who were supplementing with antioxidants (in accordance with our instructions and inclusion criteria) and those who were not supplementing with antioxidants.

\section{Discussion}

One of the key findings of the present study is the indication that an enhanced plasma antioxidant capacity in response

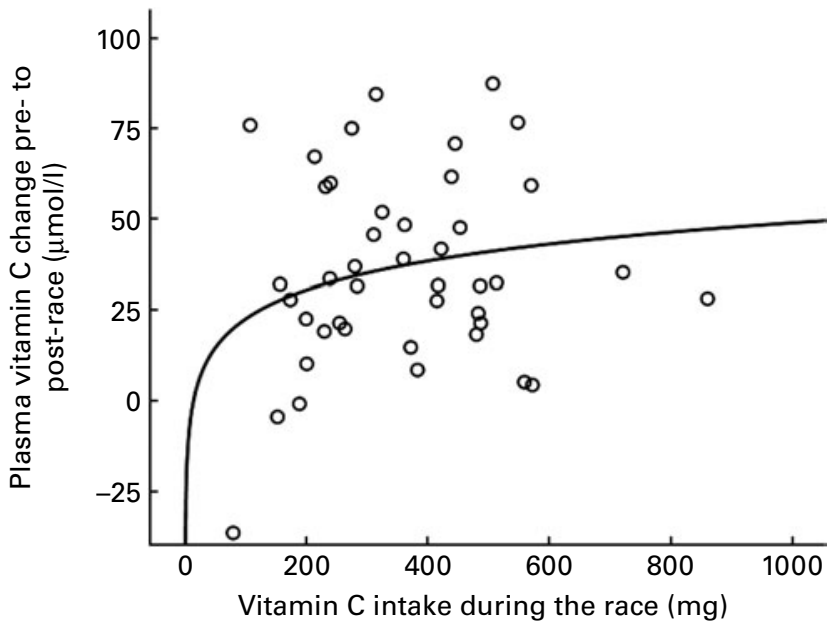

Fig. 2. Logarithmic regression of the change in the plasma vitamin $\mathrm{C}$ concentration from pre-race to post-race $v$. the vitamin $C$ intake during the Ironman race $\left(y=-12.91+8.33 \times \log (x) ; r^{2} 0.31 ; P=0.248\right)$. For details, see Discussion.

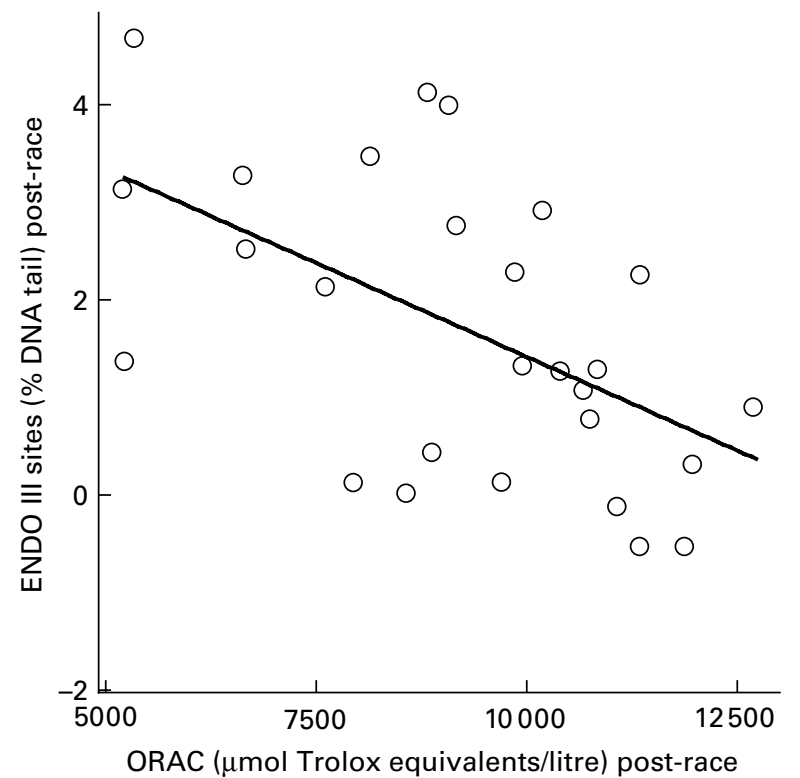

Fig. 3. Inverse correlation between the total plasma antioxidant capacity as assessed by the oxygen radical absorbance capacity (ORAC) assay and oxidative DNA damage as detected by the use of the lesion-specific enzyme endonuclease (ENDO) III immediately after the Ironman triathlon $(r-0.54$; $P<0.001)$

to the Ironman triathlon prevented oxidative DNA damage in circulating lymphocytes. Although the ad libitum intake of antioxidants during the race might have exceeded the acute demands in exogenously derived antioxidants, it might have affected plasma concentrations and, consequently, preserved antioxidant responses to a certain degree. The observed decrease in the plasma concentrations of carotenoids and $\gamma$-tocopherol $1 \mathrm{~d}$ post-race points to an additional stress generated by a low intake of these antioxidant nutrients and phytochemicals during and within the first $24 \mathrm{~h}$ after the race and an increased need for nutritive antioxidants in the early recovery phase after an acute bout of ultra-endurance exercise. Furthermore, the present results underline the importance of an adequate habitual intake of nutritive antioxidants to maintain a normal physiological antioxidant status in reference to the current recommendations ${ }^{(22)}$.

Changes of endogenous and nutritive antioxidants in response to the Ironman race, and the contribution of these antioxidants to changes in the plasma antioxidant capacity

As suggested in the literature ${ }^{(24,36)}$, a 'battery' of different measurements was applied to adequately assess the total antioxidant capacity in plasma. Actually no single test fully reflects the overall antioxidant capacity of plasma due to the different methodological principles of and associated limitations with these assays ${ }^{(24,36,37)}$. However, the time courses of the values obtained in the TEAC, FRAP and ORAC assays were similar over the entire whole monitoring period (Fig. 1), suggesting a biologically significant finding. The plasma antioxidant capacity increased significantly following the Ironman triathlon, which reflects an acute response to oxidative stress ${ }^{(36,38)}$, and remained elevated $1 \mathrm{~d}$ 
post-race ${ }^{(17,19)}$. While TEAC was found to be still increased $4 \mathrm{~d}$ after a marathon ${ }^{(38)}$, levels of the TEAC, FRAP and ORAC had returned to the pre-race values $5 \mathrm{~d}$ post-race along with oxidative stress markers in the present study. Noteworthily, we observed associations between the activities of the erythrocyte glutathione peroxidase and TEAC levels throughout the monitoring period, which may indicate a synergistic interaction between erythrocytes and the plasma antioxidant capacity ${ }^{(17)}$. There is emerging evidence that the efficient erythrocytes' antioxidant machinery, due to the erythrocytes' mobility and high cell density in the blood, protects not only their own membrane and local environment, but also contributes to the maintenance of circulatory antioxidant levels and the oxidant-scavenging ability throughout the circulation ${ }^{(37,39)}$. Furthermore, both TEAC and glutathione peroxidase activities were positively related with a variety of physiological training-related determinants ${ }^{(17)}$. Nevertheless, on the basis of these results, it is unclear whether or to which extent the training adaptations of endogenous antioxidant mechanisms (in particular, antioxidant enzymes) had an impact on the plasma antioxidant capacity.

Our findings suggest that several mechanisms contributed to the increase of the antioxidant capacity of plasma in response to the Ironman triathlon. First, in line with the previous findings ${ }^{(11,38)}$, obtained associations between changes in uric acid and those in the TEAC, FRAP and the ORAC assays ${ }^{(17,19)}$ indicate that uric acid may be responsible for the rise in the total plasma antioxidant capacity to a considerable extent. Plasma concentrations of the potent hydrophilic antioxidant uric acid are known to increase during intense exercise, with it being produced from increased purine metabolism ${ }^{(38)}$ and probably also because of impaired renal clearance ${ }^{(11)}$. Interestingly, TEAC, FRAP and uric acid increased with the athletes' performance in the Ironman race $^{(17)}$. However, as discussed previously ${ }^{(17)}$, this phenomenon is probably reflective of a higher degree of exhaustion in those athletes with a higher training- and performancestatus rather than with a specific training adaptation. Secondly, further correlations were observed between changes in FRAP and total bilirubin (that probably increased as a result of enhanced haemolysis ${ }^{(40)}$ ) and between pre- and post-race levels of ORAC and total plasma protein. The latter observation is in agreement with the results of a previous study that indicated the contribution of protein-associated thiol (sulphydryl) groups to plasma antioxidant capacity after a marathon ${ }^{(38)}$. Thirdly, the moderate correlation of the exercise-induced increase of vitamin $\mathrm{C}$ with the change of TEAC might point to a small, but significant effect of vitamin $\mathrm{C}$ on the total antioxidant capacity. Correspondingly to a study in ultra-marathon runners ${ }^{(26)}$, the immediate increase in vitamin $\mathrm{C}$ probably can be attributed to both mobilisation of ascorbic acid from the adrenal glands ${ }^{(35)}$ and/or leucocytes ${ }^{(41)}$ and consumption during the race along with the ingestion of specified and vitamin-fortified beverages and food (e.g. sports drinks and carbohydrate bars). The high variation in the intake of vitamin $C$ and, even more, of $\beta$-carotene can probably be explained by the different products used by the study participants. The lacking association between $\alpha$-tocopherol and the total antioxidant capacity could be explained by the overestimation of hydrophilic substances with the applied assays ${ }^{(24)}$.
Potential explanations for the lacking dose effects between intake of antioxidative vitamins or $\beta$-carotene during the race and their post-race plasma concentrations

No dose effect between the intake and plasma concentrations of antioxidative vitamins or $\beta$-carotene was observed based on the correlations or the group distribution into percentiles by the intake. However, based on these findings, one cannot rule out that the relatively high intake of vitamin $\mathrm{C}$ and $\alpha$-tocopherol during the race may have affected plasma concentrations (at least partly) and, consequently, might have preserved antioxidant responses to some extent. With regard to vitamin $\mathrm{C}$, the fact that the logarithmic regression of the intake during the race and the change in the plasma concentration pre-race to post-race was not significant can be taken as an indication that the average intake of $393 \mathrm{mg}$ might have exceeded the needs and/or absorption capacity (Fig. 2). The optimal bioavailability of vitamin $\mathrm{C}$ is known to be reached at an intake of $200 \mathrm{mg} / \mathrm{d}^{(42)}$. Therefore, the consumption during the race, which was nearly double-fold above this threshold (within a mean period of $10 \cdot 7 \mathrm{~h}$ ), could have influenced its plasma concentration, but probably, did not lead to a further increase ${ }^{(6)}$. Concerning $\alpha$-tocopherol, its increase in plasma levels immediately post-race is probably associated with exercise-induced changes in the lipoprotein metabolism, and might rather reflect a shift from tissue stores to plasma circulation than a response to the intake $^{(11,43)}$. An alternative or complementary explanation for the lack of obvious effects of the intake of antioxidative vitamins and carotenoids on its post-race plasma concentrations might be their metabolisation rapidly after appearance in the circulation (by scavenging RONS).

\section{Antioxidant vitamin and carotenoid status}

It is notable that the plasma concentrations of antioxidant vitamins and carotenoids of the participants were in a normal physiological range and, with the exception of $\alpha$-tocopherol (as discussed below), adequate in reference to the current recommended values ${ }^{(22)}$. Importantly, the subjects' habitual antioxidant supplementation was restricted to low dosages (according to the inclusion criteria), and no significant differences in any antioxidant parameter were observed between those who were supplemented with antioxidants and those who were not. Hence, the present results support the assumption that the requirements for vitamin $C$ and $\beta$-carotene in competitive endurance athletes, to a great if not to the full extent, can be achieved by a diversified and well-balanced $\operatorname{diet}^{(42)}$. Furthermore, the athletes' regular intake of fruits and vegetables apparently was sufficient to maintain the plasma concentrations of vitamin $\mathrm{C}$ and $\beta$-carotene above those levels which are considered as beneficial in preventing cancer and CVD throughout the study period ${ }^{(22)}$. Endurance athletes seem to meet the general recommendations for these antioxidants probably also due to their increased daily energy expenditure and food intake, provided that the nutrient density is adequate ${ }^{(42)}$, which is also supported by our FFQ data (unpublished results). Exemplarily, the mean pre-race plasma concentration of vitamin C (66.6 (SD 13.0) $\mu \mathrm{mol} / \mathrm{l})$ was beyond the level that has been observed to be sufficient to saturate immunocompetent cells $(>50 \mu \mathrm{mol} / \mathrm{l})^{(22,44)}$. 
On the other hand, although in a normal physiological range ${ }^{(22)}$, plasma $\alpha$-tocopherol concentrations of the subjects were moderate, and in agreement with a cross-sectional study in well-trained athletes ${ }^{(42)}$, high inter-individual variation among the subjects were found. However, when interpreting $\alpha$-tocopherol status in response to intense training and/or acute demanding endurance exercise, one has to bear in mind that $\alpha$-tocopherol plasma concentrations may mainly reflect mobilisation from tissue stores to plasma circulation ${ }^{(6,42)}$. Furthermore, despite an often low vitamin $\mathrm{E}$ intake ${ }^{(42)}$, welltrained athletes might not necessarily be at risk for a marginal vitamin $\mathrm{E}$ status, since certain mechanisms responsible for the incorporation of plasma $\alpha$-tocopherol into muscle cells seem to be enhanced due to training ${ }^{(6)}$. In view of the recent findings that high-dosed supplementation with vitamin $\mathrm{E}$ resulted in pro-oxidant effects after an Ironman triathlon ${ }^{(12)}$, endurance athletes should be encouraged to abstain from supra-physiological doses of vitamin E, but be encouraged to increase their dietary vitamin $\mathrm{E}$ intake. The latter requires a substantial increase in the consumption of foods that are rich in fats, such as nuts, margarine and certain oils ${ }^{(42)}$. This is supported by our (unpublished) FFQ data showing that the plasma $\alpha$-tocopherol concentration was highest (29.6 (SD 6.6) $\mu \mathrm{mol} / \mathrm{l}$ ) in the subject group that reported daily intake of nuts.

While evidence of the benefits of antioxidant supplementation in athletes in general is still scarce and inconsistent $^{(18,24)}$, the optimal bioavailability and combined action of multiple phytochemical and antioxidant compounds derived from fruits and vegetables cannot be replaced by supplementation $^{(22,36,42)}$. Finally, in the light of the postulation that RONS play important physiological roles as signalling molecules (e.g. by stimulating training adaptations in the endogenous antioxidant defences) ${ }^{(2)}$, supra-physiological doses of antioxidants may even blunt beneficial exercise-induced cellular adaptations by excessive elimination of $\operatorname{RONS}^{(3,4)}$.

\section{Early recovery responses of the antioxidant vitamins and carotenoids in the plasma}

The observation that plasma concentrations of carotenoids and tocopherols decreased to below the pre-race values $1 \mathrm{~d}$ post-race significantly, except for $\alpha$-tocopherol $(P>0.05)$ (Table 3), may indicate an increased need for antioxidant nutrients in the early recovery period after an acute bout of ultra-endurance exercise. However, contrary to the low intake of $\beta$-carotene and the moderate intake of $\alpha$-tocopherol, the consumption of vitamin $C$ within the first $24 \mathrm{~h}$ post-race (partly provided by vitamin-fortified foods and drinks) was sufficient to prevent a decrease to below the baseline plasma vitamin C levels. Similarly, Palazzetti et al. ${ }^{(45)}$ reported a drop in serum concentrations of antioxidant vitamins after 4 weeks of overloaded training, which was compensated by the intake of an antioxidant mixture containing vitamin $\mathrm{C}$ and $\alpha$-tocopherol at doses that can be provided by a diversified and well-balanced diet. The authors suggested that the antioxidant supplementation at a physiological level sufficiently helped to preserve the antioxidant system during heavy training in subjects with initially low antioxidant intakes ${ }^{(45)}$. Taken together, these results indicate that the intake of antioxidant nutrients along with a diet rich in phytochemicals after such a major competition has to be considered carefully.
Effect of antioxidant responses on oxidative stress markers and DNA damage

Another main aim of the present study was the clarification of the question whether the observed strong antioxidant responses in plasma have an influence on the markers of oxidative damage of blood lipids and blood cell compounds ${ }^{(17)}$ and endpoints of DNA stability in circulating lymphocytes ${ }^{(19)}$. Notably, plasma and blood cells are of particular importance in regulating and reflecting systemic redox status changes during and after exercise ${ }^{(37)}$. As reported previously, a transient increase of DNA strand breaks $1 \mathrm{~d}$ post-race was detected $^{(19)}$, but no indications of oxidatively damaged DNA immediately or $1 \mathrm{~d}$ post-race (as indicated by the use of lesion-specific enzymes in the single-cell gel electrophoresis assay) were observed. Importantly, negative correlations were observed between ORAC and endonuclease III-sensitive sites immediately (Fig. 3) and $1 \mathrm{~d}$ after the Ironman race ${ }^{(20)}$. Concomitant with a recent animal study ${ }^{(46)}$, where an enhanced serum antioxidative capacity prevented lymphocyte apoptosis, the present findings suggest a protective role of the increased plasma antioxidant capacity in DNA stability.

Except the correlations between the changes in vitamin $\mathrm{C}$ and TEAC, no further associations between the plasma levels of nutritive antioxidants, on the one side, and alterations in the plasma antioxidant capacity, oxidative stress markers or endpoints of DNA stability, on the other side, were observed. As a possible explanation, it is feasible to suggest that the acute oxidative stress response to an ultra-endurance exercise may exceed the capacity of exogenously consumed antioxidants. Otherwise, the lack of significant effects of especially nutritive antioxidants on exercise-induced increases of lipid peroxidation and protein oxidation markers ${ }^{(17)}$ could be due to the fact that plasma concentrations of antioxidant nutrients of all the subjects were in a normal physiological range ${ }^{(22)}$. Another important point to keep in mind is that our data are in reference to a sample cohort of young and healthy athletes with an adequate training status ${ }^{(17)}$, which is associated with an adaptive improvement of endogenous antioxidant and repair systems ${ }^{(2,5)}$. It is possible that significant effects (e.g. differences in the magnitude of oxidative stress markers) may be obtained when comparing younger $v$. older, poorly trained $v$. well-trained athletes or athletes with a lower $v$. a higher antioxidant status. As reported previously ${ }^{(17)}$, we found that even minor differences in the training status among the same group of athletes resulted in different changes in lipid peroxidation markers in response to the Ironman race, indicating that better training levels might confer enhanced protection against oxidative stress. However, on the contrary, individual differences in the plasma levels of antioxidant nutrients might have been too small to detect similar findings related to the nutritive antioxidant status. Furthermore, in this context, it is important to note the general difficulty in determining correlations among markers of DNA damage, biomarkers of oxidative stress and antioxidant parameters ${ }^{(47)}$.

\section{Methodological considerations and limitations}

Despite our efforts to address the main factors influencing the antioxidant status and requirements, we are aware of the major difficulties to tightly control all of them in a 
field-based study, e.g. to completely forbid supplementation for athletes. However, the study participants were precisely instructed to restrict from using antioxidant supplementation higher than $100 \%$ RDA (see Experimental methods). RDA (not the current dietary recommended intakes) levels as established by the US Food and Nutrition Board of the Institute of Medicine ${ }^{(21)}$ were used for the following reasons: on the one hand, antioxidant dosages up to $100 \%$ of RDA represent physiological doses. On the other hand, these RDA levels are used for labelling food and supplementation products (based on the Austrian food law), which facilitated the compliance of the study participants. As described, no effects of antioxidant supplementation were observed. Furthermore, in awareness of the obvious difficulties associated with the self-reported dietary intake data during such a competition (e.g. the athletes' ability to recall information thereafter), the food intake at each of the supply stations of the race was carefully monitored by supporters to verify the nutritional information gained from the $24 \mathrm{~h}$ dietary recalls.

\section{Implications for the antioxidant intake in ultra-endurance exercise}

The general picture that emerges from the data of the present study is that training- and acute exercise-induced adaptations in protective mechanisms including endogenous antioxidant defences counteracted severe oxidative damage of blood lipids, blood cell compounds ${ }^{(17)}$ and DNA in circulating lymphocytes $^{(18-20)}$ in response to an ultra-endurance exercise. Importantly, the increase in the total plasma antioxidant capacity played a major role, and a protective effect was apparent with regard to DNA stability. Endogenous mechanisms seem to be more important in the acute exercise-induced changes of the plasma antioxidant capacity than the impact of exogenously derived antioxidants. However, the present results indicate that the antioxidant intake during and in the early recovery period after intense and very prolonged exercise requires specific attention. Furthermore, the present results illustrate that requirements of nutritive antioxidants to maintain an adequate physiological antioxidant status in ultra-endurance athletes (in reference to recommendations), to a large, if not to the full extent, can be achieved by consumption of a balanced and well-diversified diet.

\section{Acknowledgements}

The present data are part of a research project that was funded by the Austrian Science Fund (FWF). The authors' contributions are as follows: O. N. and K.-H. W. contributed to the study design and realisation of the study; K.-H. W. and S. K. were involved in the supervision of the work; S. R., L. N., C. H., J. V., B. S. and O. N. conducted the experimental work; O. N. and S. R. performed the statistical analysis; O. N. wrote the manuscript. Furthermore, the authors would like to thank Anna Chalopek and Norbert Kern for their assistance in the data collection and chemical analysis, and all the study participants for their efforts. None of the authors has a conflict of interest to declare.

\section{References}

1. Powers SK \& Jackson MJ (2008) Exercise-induced oxidative stress: cellular mechanisms and impact on muscle force production. Physiol Rev 88, 1243-1276.

2. Ji LL (2008) Modulation of skeletal muscle antioxidant defense by exercise: role of redox signaling. Free Radic Biol Med 44, $142-152$.

3. Gomez-Cabrera MC, Domenech E, Romagnoli M, et al. (2008) Oral administration of vitamin $\mathrm{C}$ decreases muscle mitochondrial biogenesis and hampers training-induced adaptations in endurance performance. Am J Clin Nutr 87, 142-149.

4. Ristow M, Zarse K, Oberbach A, et al. (2009) Antioxidants prevent health-promoting effects of physical exercise in humans. Proc Natl Acad Sci U S A 106, 8665-8670.

5. Vina J, Gomez-Cabrera MC \& Borras C (2007) Fostering antioxidant defences: up-regulation of antioxidant genes or antioxidant supplementation? Br J Nutr 98, Suppl. 1, S36-S40.

6. Margaritis I \& Rousseau AS (2008) Does physical exercise modify antioxidant requirements? Nutr Res Rev 21, 3-12.

7. Millet GP, Dreano P \& Bentley DJ (2003) Physiological characteristics of elite short- and long-distance triathletes. Eur J Appl Physiol 88, 427-430.

8. Jeukendrup AE, Jentjens RL \& Moseley L (2005) Nutritional considerations in triathlon. Sports Med 35, 163-181.

9. Lee IM, Hsieh CC \& Paffenbarger RS Jr (1995) Exercise intensity and longevity in men. The Harvard Alumni Health Study. JAMA 273, 1179-1184.

10. Knez WL, Coombes JS \& Jenkins DG (2006) Ultra-endurance exercise and oxidative damage: implications for cardiovascular health. Sports Med 36, 429-441.

11. Mastaloudis A, Morrow JD, Hopkins DW, et al. (2004) Antioxidant supplementation prevents exercise-induced lipid peroxidation, but not inflammation, in ultramarathon runners. Free Radic Biol Med 36, 1329-1341.

12. Nieman DC, Henson DA, McAnulty SR, et al. (2004) Vitamin E and immunity after the Kona Triathlon World Championship. Med Sci Sports Exerc 36, 1328-1335.

13. Knez WL, Jenkins DG \& Coombes JS (2007) Oxidative stress in half and full ironman triathletes. Med Sci Sports Exerc 39, 283-288.

14. Nieman DC, Henson DA, McAnulty SR, et al. (2002) Influence of vitamin $\mathrm{C}$ supplementation on oxidative and immune changes after an ultramarathon. J Appl Physiol 92, 1970-1977.

15. Neubauer O, König D \& Wagner KH (2008) Recovery after an Ironman triathlon: sustained inflammatory responses and muscular stress. Eur J Appl Physiol 104, 417-426.

16. König D, Neubauer O, Nics L, et al. (2007) Biomarkers of exercise-induced myocardial stress in relation to inflammatory and oxidative stress. Exerc Immunol Rev 13, 15-36.

17. Neubauer O, König D, Kern N, et al. (2008) No indications of persistent oxidative stress in response to an ironman triathlon. Med Sci Sports Exerc 40, 2119-2128.

18. Reichhold S, Neubauer O, Ehrlich V, et al. (2008) No acute and persistent DNA damage after an Ironman triathlon. Cancer Epidemiol Biomarkers Prev 17, 1913-1919.

19. Reichhold S, Neubauer O, Hoelzl C, et al. (2009) DNA damage in response to an Ironman triathlon. Free Radic Res 43, $753-760$.

20. Neubauer O, Reichhold S, Nersesyan A, et al. (2008) Exerciseinduced DNA damage: is there a relationship with inflammatory responses? Exerc Immunol Rev 14, 51-72.

21. Food and Nutrition Board/Institute of Medicine (2000) Dietary Reference Intakes for Vitamin C, Vitamin E, Selenium, and Carotenoids. Washington, DC: National Academies Press.

22. DACH (2000) Referenzwerte für die Nährstoffzufuhr, Deutsche Gesellschaft für Ernährung, Österreichische Gesellschaft 
für Ernährung, Schweizerische Gesellschaft für Ernährungsforschung, Schweizerische Vereinigung für Ernährung; Umschau Braus, Frankfurt am Main (Reference values for nutrient intake, German Nutrition Society, Austrian Nutrition Society, Swiss Society of Food Research, Swiss Association for Nutrition, Umschau Braus, Frankfurt am Main).

23. Ramel A, Wagner KH \& Elmadfa I (2004) Plasma antioxidants and lipid oxidation after submaximal resistance exercise in men. Eur J Nutr 43, 2-6.

24. Knasmuller S, Nersesyan A, Misik M, et al. (2008) Use of conventional and -omics based methods for health claims of dietary antioxidants: a critical overview. Br J Nutr 99, Suppl. 1, ES3-ES52.

25. Tomasch R, Wagner KH \& Elmadfa I (2001) Antioxidative power of plant oils in humans: the influence of alpha- and gamma-tocopherol. Ann Nutr Metab 45, 110-115.

26. Benzie IF \& Strain JJ (1996) The ferric reducing ability of plasma (FRAP) as a measure of "antioxidant power": the FRAP assay. Anal Biochem 239, 70-76.

27. Benzie IF \& Strain JJ (1999) Ferric reducing/antioxidant power assay: direct measure of total antioxidant activity of biological fluids and modified version for simultaneous measurement of total antioxidant power and ascorbic acid concentration. Methods Enzymol 299, 15-27.

28. Ou B, Hampsch-Woodill M \& Prior RL (2001) Development and validation of an improved oxygen radical absorbance capacity assay using fluorescein as the fluorescent probe. J Agric Food Chem 49, 4619-4626.

29. Huang D, Ou B, Hampsch-Woodill M, et al. (2002) Highthroughput assay of oxygen radical absorbance capacity (ORAC) using a multichannel liquid handling system coupled with a microplate fluorescence reader in 96-well format. J Agric Food Chem 50, 4437-4444.

30. Wagner KH, Reichhold S, Knasmuller S, et al. (2010) Does an Ironman triathlon consistently influence oxidative stress, antioxidant status and DNA stability? Toxicology (In the Press).

31. Tice RR, Agurell E, Anderson D, et al. (2000) Single cell gel/comet assay: guidelines for in vitro and in vivo genetic toxicology testing. Environ Mol Mutagen 35, 206-221.

32. Collins AR, Duthie SJ \& Dobson VL (1993) Direct enzymic detection of endogenous oxidative base damage in human lymphocyte DNA. Carcinogenesis 14, 1733-1735.

33. Dill DB \& Costill DL (1974) Calculation of percentage changes in volumes of blood, plasma, and red cells in dehydration. J Appl Physiol 37, 247-248.
34. Shaskey DJ \& Green GA (2000) Sports haematology. Sports Med 29, 27-38.

35. Gleeson M, Robertson JD \& Maughan RJ (1987) Influence of exercise on ascorbic acid status in man. Clin Sci (Lond) 73, 501-505.

36. Prior RL \& Cao G (1999) In vivo total antioxidant capacity: comparison of different analytical methods. Free Radic Biol Med 27, 1173-1181.

37. Nikolaidis MG \& Jamurtas AZ (2009) Blood as a reactive species generator and redox status regulator during exercise. Arch Biochem Biophys 490, 77-84.

38. Liu ML, Bergholm R, Makimattila S, et al. (1999) A marathon run increases the susceptibility of LDL to oxidation in vitro and modifies plasma antioxidants. Am J Physiol 276, E1083-E1091.

39. Buehler PW \& Alayash AI (2005) Redox biology of blood revisited: the role of red blood cells in maintaining circulatory reductive capacity. Antioxid Redox Signal 7, 1755-1760.

40. Suzuki K, Peake J, Nosaka K, et al. (2006) Changes in markers of muscle damage, inflammation and HSP70 after an Ironman triathlon race. Eur J Appl Physiol 98, 525-534.

41. Viguie CA, Frei B, Shigenaga MK, et al. (1993) Antioxidant status and indexes of oxidative stress during consecutive days of exercise. J Appl Physiol 75, 566-572.

42. Rousseau AS, Hininger I, Palazzetti S, et al. (2004) Antioxidant vitamin status in high exposure to oxidative stress in competitive athletes. Br J Nutr 92, 461-468.

43. Mastaloudis A, Leonard SW \& Traber MG (2001) Oxidative stress in athletes during extreme endurance exercise. Free Radic Biol Med 31, 911-922.

44. Levine M, Conry-Cantilena C, Wang Y, et al. (1996) Vitamin C pharmacokinetics in healthy volunteers: evidence for a recommended dietary allowance. Proc Natl Acad Sci U S A 93, 3704-3709.

45. Palazzetti S, Rousseau AS, Richard MJ, et al. (2004) Antioxidant supplementation preserves antioxidant response in physical training and low antioxidant intake. Br J Nutr 91, $91-100$.

46. Avula CP, Muthukumar AR, Zaman K, et al. (2001) Inhibitory effects of voluntary wheel exercise on apoptosis in splenic lymphocyte subsets of C57BL/6 mice. J Appl Physiol 91, 2546-2552.

47. Dotan Y, Lichtenberg D \& Pinchuk I (2004) Lipid peroxidation cannot be used as a universal criterion of oxidative stress. Prog Lipid Res 43, 200-227. 